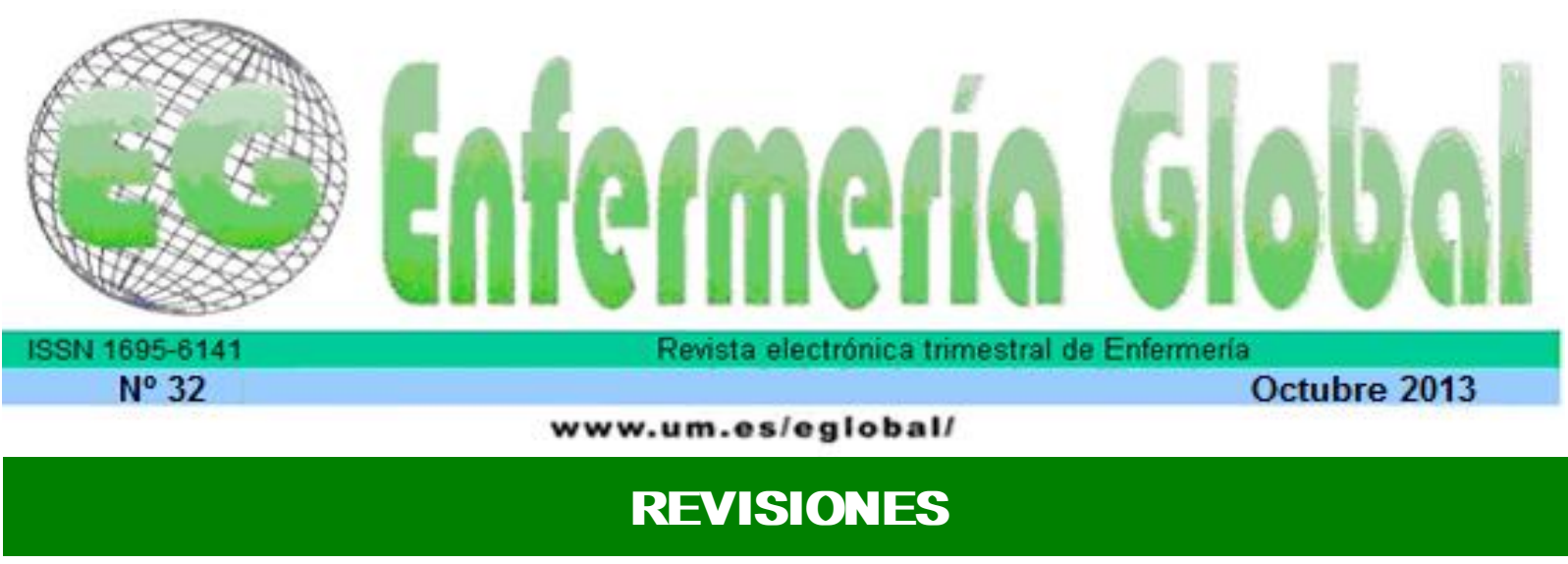

\title{
La filosofía de Patricia Benner y la práctica clínica
}

Review of Patricia Benner's philosophy in clinical practice

\section{${ }^{*}$ Carrillo Algarra, Ana Julia ${ }^{* *}$ García Serrano, Lucila ${ }^{* * *}$ Cárdenas Orjuela, Claudia Marcela ${ }^{* * *}$ Díaz Sánchez, Ingrid Rocío ${ }^{* * * * *}$ Yabrudy Wilches, Nataly}

*Enfermera, Magister en Administración en Salud. Profesora Titular de la Facultad de Enfermería. Email: ajcarrillo@fucsalud.edu.co ${ }^{* *}$ Enfermera, Magister en Educaciòn. Profesora Titular de la Facultad de Enfermería. *** Estudiante de Enfermería. Fundación Universitaria de Ciencias de la Salud. Bogotá-Colombia.

Palabras clave: Competencia clínica; Enfermería holística; Enfermería de practica avanzada; Ética en enfermería; Filosofía en enfermería; Patricia Benner.

Keywords: Clinical competence; Holistic nursing; Advanced practice nursing; Ethics nursing; Philosophy nursing; Patricia Benner

\section{RESUMEN}

Con el objetivo de explorar la aplicación de la filosofía de Patricia Benner en el ámbito clínico se realizó una búsqueda de la literatura científica inicialmente con vigencia mínima de 5 años, encontrándose durante este proceso escasa literatura, por lo cual surgió la necesidad de ampliar el período e incluir desde 1984 hasta el 2010. Se encontraron 90 artículos, de los cuales se seleccionaron 52. En el desarrollo del presente artículo se establecen las competencias que requiere el profesional de enfermería durante la práctica clínica, partiendo de los planteamientos de Patricia Benner quien modifica los niveles de adquisición de habilidades de los hermanos Dreyfus con el fin de aplicarlos a enfermeria en el área clínica; adicionalmente se relacionan con los patrones del conocimiento descritos por Barbara Carper y el perfil que debe tener la enfermera(0) que cuida pacientes que por su edad o condición clinica se encuentran en las areas de urgencias, unidad de cuidados intensivos y pediatria.

\section{ABSTRACT}

In order to explore how to apply Patricia Benner's philosophy on the clinical field, a research was done about the scientific literature, initially with a five year range. However, little material was found on that field and for this reason it was decided to increase the range to a period ranging from 1984 to 2010. 90 articles were found and 52 of them were selected. 
This article establishes the skills that a professional nurse needs during the clinical practice, based on Patricia Benner's approach that modified the Dreyfus brothers' skill acquisition levels in order to apply them to nursery in the clinical area; additionally, it is related to the knowledge patterns described by Barbara Carper and the profile that a nurse must have in order to take care of patients who, either due to their age or clinical condition, are located on the ICU or pediatrics.

\section{INTRODUCCIÓN}

La enfermera(o) que desarrolla su labor en el área asistencial, cada vez que afronta una situación realiza un aprendizaje que puede generarse por elementos de trasformación o por repetición ${ }^{1}$ creando habilidades y destrezas que solo son propias de la práctica clínica. La enfermera(o) debe crear un ambiente de organización, con la finalidad de ejercer una práctica clínica en plenitud y con éxito, ejecutando su función de líder ${ }^{2}$.

En el área de enfermería y de la salud en general, se requiere gran capacidad cognitiva, de resolución de problemas, pero fundamentalmente para relacionarse con otras personas; la enfermera(o) se ve como un individuo que debe poseer grandes capacidades para pensar y habilidad para realizar actividades, pero en ocasiones se deja de lado su parte espiritual, la ética y la moral, que forman parte fundamental de la competencia "el saber ser", es de allí donde deriva cómo hace elecciones, puesto que la ética solidaria establece puntos de partida para retomar el valor de las elecciones profesionales ${ }^{3,4}$.

Este artículo tuvo como objetivo explorar la aplicación de la filosofía de Patricia Benner en el ámbito clínico. Se parte de las propuestas de esta autora acerca del cuidado de enfermería, de las competencias y etapas de la formación de la enfermera hasta alcanzar la experticia ${ }^{5}$. Finalmente, se proponen tres perfiles que señalan las diversas características y atributos que debe tener la enfermera en el cuidado de los pacientes que se encuentran en las áreas de urgencias, cuidado crítico y pediatría.

\section{METODOLOGÍA}

Se realizó una revisión bibliográfica, en la cual las unidades de análisis fueron artículos científicos publicados entre el 1984 y 2010 en español, inglés y portugués en las bases de datos Scielo, Ebsco, Elsevier, Nursing Ovied, Dialnet, Hinary, Sciencedirect y Dynamet. Se utilizaron los términos Patricia Benner, competencias de enfermería, cuidado de enfermería y perfil de la enfermera en área de urgencias, unidad de cuidados intensivos y pediatría; encontrándose 90 artículos de los cuales se seleccionaron 52 que respondían al objetivo planteado.

\section{El cuidado y las competencias de enfermería en el área clínica}

La esencia de enfermería es velar por la salud y el bienestar del paciente que se sintetiza en una acción primordial: "Cuidar". Esta labor la define Busquets ${ }^{4}$ como: "Cuidar es acompañar a la persona en sus experiencias de vida. Por ello enfermería trata de planificar la atención desde esta perspectiva... Cuidar es cuidar a la persona concreta planteando alternativas a la cosificación y consideración del paciente y su situación como un problema a resolver"4. Se debe partir de ello para tener como resultado la satisfacción de la persona con relación al cuidado que recibe por parte de la enfermera(o) en todos sus contextos. 
Entre las diversas definiciones de cuidado se encuentra que "Cuidado también se refiere a dedicación, cuyo comportamiento, apariencia, formación moral e intelectual son de buen gusto (hablando de la persona), es la atención, el comportamiento cauteloso, el fervor, la devoción dedicada a alguien o algo..." 6 . Por esta razón el sujeto de inspiración del arte del cuidado es el paciente y como tal necesita de la atención de una enfermera(o) que al formarse competentemente estará en la capacidad de brindarle una respuesta emocional, fisiológica y oportuna a las dificultades que probablemente irà presentando en su estancia intrahospitalaria.

En este contexto, el proceso de formación de enfermería implica varias fases ya que debe estar basado en el desarrollo de diferentes competencias que constituyen la base fundamental para generar un excelente profesional de enfermería; la formación debe ser integral puesto que se educa para realizar tareas específicas y desarrollar las competencias en el sujeto cuidador desde el ser, el saber y saber hacer. Todas son importantes y dependiendo de la situación de enfermería se deben poner en práctica unas más que otras e independientemente de la actividad siempre deben estar presentes en cualquier acción que realiza la enfermera(0) ${ }^{6}$. La formación en enfermería permite crear bases sólidas para el desempeño del profesional, es un proceso de maduración que aspira a promover el avance del ser humano hacia una vida personal social, cognitiva, creativa, constructiva y productiva ${ }^{6}$.

La práctica clínica supone tanto habilidades como conocimientos que deben ser puestos en práctica para la prestación de cuidados de calidad que serán evaluados según la satisfacción del paciente y que la enfermera debe saber relacionar en el momento del actuar pues es trabajar con lo aprendido en el pregrado, es decir, con conocimiento científico al cual se suma la destreza que debe tener para ejecutar la acción en sí. La práctica clínica señala fundamentalmente algunos componentes a los cuales la enfermera le apunta;el primero es la persona o paciente quien justifica el porqué de la profesión, el segundo la esencia del ser de enfermería, que son los cuidados brindados con excelente calidad ya sea preventivo, curativo o paliativo dependiendo del tipo de paciente ${ }^{7}$.

En la práctica diaria el profesional de enfermería tiene un rol definido como sujeto cuidador, los cuidados varían dependiendo del tipo de paciente y el actuar obedece a las diferentes situaciones que se presenten, pero en general se realizan funciones que dan lugar a acciones autónomas, ágiles, responsables, eficaces y eficientes que caracterizan a un profesional de enfermería líder de un servicio que es coordinado por el profesional de enfermería ya experto ${ }^{7}$.

Partiendo del "ser" como aquella cualidad que se atribuye a sí mismo en un ambiente determinado, con características únicas, irrepetibles e insustituibles ${ }^{8}$; Hegel citado por Fernandez lo denomina como el comienzo de la ciencia, es decir, que el ser es el único determinante para el desarrollo de actividades y la creación de nuevas estrategias de crecimiento como organismo biopsicosocial ${ }^{9}$.

Los conceptos anteriores llevan a la convicción de que el individuo es imposible de separar en sus dimensiones, ya que no es fácil establecer cuáanto influye una sobre las otras o cuánto dependen la una de las otras frente al proceso salud-enfermedad, se debe aprehender y comprender la complejidad de su atención integral en salud en aras de mejorar su calidad de vida ${ }^{10}$. 
Para la enfermera(o) su razón de ser es el cuidado, su función es ver al individuo como un todo buscando su armonía y equilibrio, abordando una realidad con un modelo de cuidado de enfermería en busca de una aplicación del cuidado integral ${ }^{11}$; la responsabilidad de enfermería es fundamentar la teoría que guía su práctica, aquí es importante resaltar que Leonardo da Vinci, citado por León ${ }^{12}$, planteó que la práctica sin la teoría es como el hombre que sale a la mar sin mapa, es un barco sin timón. Con esto, se afirma que durante el proceso de enfermería se debe brindar una atención integral que reúna y cumpla con los requerimientos del individuo como ser holístico ${ }^{10}$. En enfermería este aspecto es relevante por lo que se resalta en la teoría propia. Por ejemplo, Patricia Benner ${ }^{11}$ citada por Brykczynski ha escrito sobre el desarrollo del conocimiento en una disciplina práctica, con base en la propuesta de niveles de formación de los hermanos Dreyfus ${ }^{11}$. En el presente articulo se retoma la clasificación expuesta por Benner ${ }^{11}$ y se relaciona con los patrones del conocimiento de Carper ${ }^{13}$,citada por Durán y el sociopolítico de White ${ }^{14}$, citado por Persegona, donde se tienen en cuenta las cuatro dimensiones del ser humano con la concepción de la persona como un ser integral.

\section{De novata a experta}

Importantes autoras como Patricia Benner han influenciado la creciente tendencia de conceptuar la ética, siendo una forma de conocimiento contextual incorporado en la experticia de la enfermería ${ }^{15,16}$. Lo anterior, por ser un elemento tranversal en el actuar y en el desarrollo del profesional. Así en los principales conceptos expresados por Benner ${ }^{11}$, quien retoma del modelo de Dreyfus ${ }^{17}$ citado por Peña "Con la experiencia y la maestría se trasforma la habilidad" ${ }^{17}$, plantea que todas las situaciones prácticas son mas complejas, por lo cual en su obra expone los supuestos teóricos que enmarcan la disciplina enfermera: enfermería, persona, salud y entorno.

Según Nelson ${ }^{15}$, quien cita la teoría propuesta por los hermanos Dreyfus ${ }^{15}$ en torno a la experticia y la no moral, en la cual los seres humanos no solo acuden en la vida diaria a principios para enfrentar situaciones morales dilemáticas, también se apoyan en el conocimiento de una gran cantidad de ejemplos acumulados a partir de la experiencia y con los cuales fijan un determinado curso de acción ${ }^{15}$. Esta es una de las principales fuentes teóricas de Benner ${ }^{11}$. En el modelo denominado "Adquisición y desarrollo de habilidades y competencias" los hermanos Dreyfus desarrollaron su método a partir del estudio de la actuación de pilotos en situación de emergencia y de jugadores de ajedrez, para identificar el grado de destreza de los mismos ${ }^{17}$. Observaron los niveles en la adquisición de habilidades y cómo las personas aprenden, con base en ello han ofrecido un modelo de experiencia sobre cómo un individuo adquiere experticia progresivamente y lo describen en la siguiente clasificación:

- Principiante: se utiliza la experiencia específica.

- Principiante avanzado: se utiliza el pensamiento analítico basado en normas de la instituciòn.

- Competente: el estudiante percibe que toda la formación es importante.

- Eficiente: se pasa de ser un observador externo a tener una posición de implicación total. 
- Experto: valora los resultados de la situación ${ }^{17}$.

En la etapa novata una persona sigue unas reglas que son independientes del contexto y no siente ninguna responsabilidad por cualquier otra cosa que seguir las reglas. La competencia se desarrolla después de haber vivido considerables experiencias, que permiten a los individuos utilizar la intuición en la toma de decisiones; esa experiencia se caracteriza por fluidez o rendimiento que se da automáticamente y ya no depende del conocimiento explícito ${ }^{17,18}$.

Kirschner ${ }^{19}$ define la competencia como: "El conjunto de conocimientos y capacidades que las personas tienen a su disposición y que pueden utilizar de manera eficiente y eficaz para alcanzar ciertas metas en una amplia variedad de contextos y situaciones"19. En principio una competencia está integrada por dos dimensiones: conocimientos denominada saber y habilidades que corresponde al hacer, pero hay una tercera dimensión que es quizá la más importante y es la que corresponde al $\operatorname{ser}^{19}$.

Las diferencias en la adquisición de habilidades se han investigado sobre la base de datos demográficos, como la preparación educativa, el trabajo que genera experiencia, el tipo de programa educativo, la enseñanza, la clínica y el desarrollo profesional, centrado en el currículo ${ }^{19}$. Pero el complejo proceso de adquisición de competencias según Dumas ${ }^{20}$, incluye la integración de diferentes planos, los cognitivos, afectivos y conductuales ${ }^{18,20}$.

Los orientadores del aprendizaje en Enfermería requieren estar preparados en lo disciplinar, lo profesional y en pedagogía, simultáneamente, su práctica debe estar basada en la evidencia, con éstas caracteristicas los educadores del futuro son eficaces y tienen las competencias necesarias para facilitar el aprendizaje en un entorno cada vez más complejo de cuidado de la salud ${ }^{21}$, guiar a los estudiantes a su nivel más alto de pensamiento independiente y de competencia puede lograrse mejor a través de los métodos de enseñanza que estimulan el pensamiento, hacen hincapié en la resolución de problemas y afectan a la motivación para aprender ${ }^{22}$.

La Filosofia de Patricia Benner ${ }^{11}$ muestra el proceso que la enfermera(o) atraviesa desde recién graduada(o) hasta que se especializa en un área determinada. Durante este proceso van surgiendo una serie de cambios de conducta; se van adquiriendo habilidades que hacen que el desempeño profesional sea cada vez de mejor calidad $^{23}$.

Robinson ${ }^{24}$ señala cuatro etapas de adquisición de habilidades intelectuales y motoras que se ponen en práctica en el quehacer diario de la profesión, las describe así: la enfermera novata, la cual comienza a relacionar el conocimiento práctico con el teórico frente a situaciones de baja complejidad desarrollando así un plan de atención básica. La enfermera competente una y la dos, en la cual la primera hace referencia a la profesional que se encuentra en la etapa en la cual domina actividades básicas, pone en práctica experiencias del pasado para resolver situaciones actuales, el núcleo familiar entra a ser parte del proceso de enfermería; y la segunda, se basa en experiencias previas dando soluciones rápidas a situaciones no planificadas con eficiencia y eficacia. La enfermera ya es competente en cuanto a analizar, predecir y actuar. Por último, la enfermera experta es capaz de resolver problemas de alta complejidad, tiene una base intuitiva, genera pensamiento crítico y autocritico, mantiene un equipo de trabajo en mejora continua, dando como resultado la 
prestación de un servicio de alta calidad y satisfacción para los pacientes y familiares $^{24}$.

En la siguiente tabla se relaciona la clasificación del modelo de Dreyfus ${ }^{17}$, con las etapas de adquisición de habilidades adaptado por Benner ${ }^{11}$, para Enfermería, donde se presentan las características correspondientes de la enfermera en la práctica clínica.

Tabla No. 1. Clasificación de Benner: Conceptos principales y definiciones de las etapas de la enfermera en la clínica.

\begin{tabular}{|c|c|c|c|c|}
\hline ante & $\begin{array}{l}\text { incipiante } \\
\text { ranzada }\end{array}$ & ;ompetente & Eficiente & Experta \\
\hline $\begin{array}{l}\text { La persona se } \\
\text { enfrenta a una } \\
\text { nueva } \\
\text { situación. Este } \\
\text { nivel Benner } \\
\text { indica que } \\
\text { podría } \\
\text { aplicarse a una } \\
\text { enfermera } \\
\text { inexperta o la } \\
\text { experta cuando } \\
\text { se desempeña } \\
\text { por primera vez } \\
\text { en un área o en } \\
\text { alguna } \\
\text { situación } \\
\text { conocida no } \\
\text { previamente } \\
\text { por ella. }\end{array}$ & $\begin{array}{l}\text { En este nivel la } \\
\text { enfermera } \\
\text { después de } \\
\text { haber adquirido } \\
\text { experiencias se } \\
\text { siente con } \\
\text { mayor } \\
\text { capacidad de } \\
\text { plantear una } \\
\text { situación } \\
\text { clínica } \\
\text { haciendo un } \\
\text { estudio } \\
\text { completo de } \\
\text { ella } \\
\text { posteriormente } \\
\text { demostrara sus } \\
\text { capacidades y } \\
\text { conocerá todo } \\
\text { lo que esta } \\
\text { exige. }\end{array}$ & $\begin{array}{lr}\text { Se } & \text { es } \\
\text { competente } & \\
\text { cuando } & \text { la } \\
\text { enfermera } & \\
\text { posee } & \text { la } \\
\text { capacidad de } \\
\text { imitar lo que } \\
\text { hacen los } \\
\text { demás a partir } \\
\text { de situaciones } \\
\text { reales; } \\
\text { enfermera la } \\
\text { empieza } \\
\text { reconocer los } \\
\text { patrones para } \\
\text { así priorizar su } \\
\text { atención, como } \\
\text { también es } \\
\text { competente } \\
\text { cuando elabora } \\
\text { una } \\
\text { planificación } \\
\text { estandarizada } \\
\text { por si misma. }\end{array}$ & $\begin{array}{lr}\text { La enfermera } \\
\text { percibe la } \\
\text { situación de } \\
\text { manera integral } \\
\text { y reconoce sus } \\
\text { principales } \\
\text { aspectos ya } \\
\text { que posee un } \\
\text { dominio } \\
\text { intuitivo sobre } \\
\text { esta; se siente } \\
\text { más segura de } \\
\text { sus } \\
\text { conocimientos } \\
\text { y destrezas, y } \\
\text { esta mas } \\
\text { implicada con } \\
\text { el paciente y su } \\
\text { familia. }\end{array}$ & $\begin{array}{lr}\text { La enfermera } \\
\text { Posee } & \text { un } \\
\text { completo } & \\
\text { dominio } & \\
\text { intuitivo } & \text { que } \\
\text { genera ra } & \text { la } \\
\text { capacidad de } \\
\text { identificar un } \\
\text { problema sin } \\
\text { perder tiempo } \\
\text { en soluciones } \\
\text { alternativas, así } \\
\text { mismo, } \\
\text { reconoce } \\
\text { patrones } \\
\text { conoce a sus } \\
\text { pacientes } \\
\text { aceptando sus } \\
\text { necesidades } \\
\text { sin importar } \\
\text { que esto le } \\
\text { implique } \\
\text { planificar y/o } \\
\text { modificar } \\
\text { plan el } \\
\text { cuidado. de }\end{array}$ \\
\hline
\end{tabular}

Fuente: Brykczynski, 2005.

A efectos del presente artículo se tomará la clasificación de Patricia Benner ${ }^{11}$, descrita en la tabla número uno por tener aplicabilidad a los profesionales de enfermería en el ámbito clínico, en el cual afirma que la práctica refuerza, fija el conocimiento y hace énfasis en la investigación científica basada en el conocimiento práctico, lo que hace referencia al "saber práctico" ${ }^{11-25}$.

Enfermería se configura como una disciplina profesional, por tanto su desarrollo ha ocurrido a partir de la actividad práctica en el cuidado de los seres humanos. El Consejo Internacional de Enfermería ${ }^{26}$ (CIE) define la Enfermería como la disciplina que "abarca los cuidados, autónomos y en colaboración, que se prestan a las personas de todas las edades, familias, grupos y comunidades, enfermos o sanos, en 
todos los contextos, e incluye la promoción de la salud, la prevención de la enfermedad, y los cuidados de los enfermos, discapacitados, y personas moribundas. Funciones esenciales de la enfermería son la defensa, el fomento de un entorno seguro, la investigación, la participación en la política de salud y en la gestión de los pacientes y los sistemas de salud, y la formación" ${ }^{26}$. Esta condición es visualizada al percibir a los trabajadores enfermeros como los profesionales del cuidado ${ }^{27}$.

Según Landeros ${ }^{28}$, la Enfermería "observa el fenómeno, ya sea casual o incidental, se analizan sus dimensiones, se buscan en la literatura antecedentes, investigaciones y reflexiones respecto al tema, se razona, se adquiere la experiencia de aprendizaje, se reproduce el fenómeno y finalmente cambia la conducta, la cual se puede compartir a través del ejemplo de nuestra actitud o a través de la comunicación verbal", ${ }^{28}$ lo cual reafirma que los componentes básicos de enfermería como disciplina son definitivamente la práctica, la teoría y la investigación ${ }^{27}$ pero esta profesión, desde sus orígenes, se ha basado en la práctica.

Con lo anterior se observa que en la formación disciplinar el conocimiento llevado a la práctica es el eje fundamental para el desarrollo de habilidades y destrezas del profesional de enfermería ${ }^{25}$, en concordancia con lo planteado por Carper $^{28}$, citada por Landeros quién definió que "el cuerpo de conocimientos que da soporte a Enfermería se expresa por medio de características externas e internas que dejan ver la manera como se piensa de un fenómeno"28. Lo anterior hace referencia a los patrones del conocimiento, centrando su atención en el significado de la disciplina de enfermería. Carper ${ }^{28}$ "determinó que la ciencia enfermera se nutre de cuatro fuentes o patrones básicos de conocimiento: empírico, ético, personal y estético"13-28. El conocimiento empírico representa a la ciencia entendida de forma tradicional que suele aparecer en forma de principios, leyes y teorías de aplicación general. El patrón ético se relaciona con los deberes, valores y derechos que rigen la práctica. El conocimiento personal hace referencia a la construcción del "yo" en relación con el "otro" y se forja en el entorno de la experiencia interactiva con los demás. Por último, el patrón de conocimiento estético está representado por el significado subjetivo de las acciones, comportamientos, actitudes e interacciones del profesional de enfermería en su relación de ayuda al paciente"13-28. Carper, expresa que mediante el desarrollo de los patrones del conocimiento, logra crear una visión amplia frente al individuo como ser integral, partiendo desde su ser hasta su estética no solo como figura física sino complemento y armonía de si mismo ${ }^{28}$.

Carper $^{28}$ sugiere que una razón alternativa para la falta de reconocimiento de la importancia del arte de la enfermería es que en el pasado "la definición del término arte ha sido excesiva y limita indebidamente" ${ }^{28,29}$, toma como base para argumentar una consideración más amplia de condiciones, situaciones y experiencias en enfermería que pueden llamarse propiamente estético. También se "aduce que el cuidado es el ingrediente más importante en el proceso curativo, ya que las acciones y decisiones de cuidado hacen la real diferencia en cuanto a consecuencias curativas efectivas $^{30}$. Situaciones de grave enfermedad o de proximidad a la muerte, piden hacer a un lado el significado instrumental del cuidado y poner en el centro el aspecto más necesario y central de éste: el arte de estar completamente presente para la otra persona" ${ }^{31}$.

El saber práctico parte de la formación empírica de la enfermera, permitiendo el desarrollo de la investigación en enfermería lo que ha favorecido y enriquecido notablemente la teoría y la práctica disciplinar ${ }^{8}$. De esto último se estiman los 
espacios o ámbitos en los que se favorece la adquisición de experiencias puesto que la enfermera no solo labora en un área urbana, también puede desenvolverse en un campo rural donde se verá involucrada en situaciones complejas donde se presentaran diversas dificultades a las cuales debe darle su respectiva solución de manera eficaz y eficiente; del mismo modo estas experiencias le generan curiosidad la cual la llevará a la investigación para crear así un hábito en su práctica clínica ${ }^{32}$. Entonces, es así como los entornos son de tal importancia para la enfermera que desde que se es aprendiz contribuye en su formación permitiendo que exista más coherencia entre lo que se revisa en la literatura y lo que se aprende a diario en un campo determinado ${ }^{15}$.

Heidegger, citado por Molina ${ }^{33}$, plantea que el saber que se obtiene de una situación donde se comparten habilidades, hábitos y conocimientos no existe en los libros,sino más bien en las experiencias laborales de los enfermeros asistenciales ${ }^{33}$. Teniendo en cuenta la anterior definición se encuentra que la teoría de Benner ${ }^{11}$ es ejecutada en el día a día por los estudiantes y profesionales de enfermería identificando problemáticas y necesidades de la persona, familia y comunidad en busca continua de soluciones que se ajusten a la praxis de enfermería. En el actuar diario se adquieren una serie de habilidades con relación a la atención del paciente o de la persona enferma en cualquier etapa de su vida ${ }^{34}$.

El enfoque de Benner ${ }^{35}$ de principiante a experto es el de una formación por competencias, las cuales se verán reflejadas en el quehacer de enfermería o en el crecimiento personal y profesional. Existen destrezas que solo se podrán fijar al saber práctico en este ámbito, donde el profesional entra en contacto directo con el paciente. De esta manera también se debe obtener la facilidad de aplicar los valores, aptitudes y actitudes ya que el desarrollo personal se basa en estos tres grandes factores que evidencian la extraordinaria capacidad que tiene el enfermero para la solución de cualquier conflicto o problema que pueda presentarse en el área clínica ${ }^{35}$.

En el ámbito clínico se protagoniza un papel en el que se cuida la salud de un ser humano que lo necesita. Allí el profesional de enfermería demuestra que tan competente es al brindar un cuidado integral a la persona hospitalizada pues ella se sentirá satisfecha y su estadía en el hospital no se convertirá en una experiencia desagradable. Se debe ser estricto en cuanto al conocimiento científico de las intervenciones y su respectiva justificación, puesto que se enfrentan situaciones que requieren del saber inmediato y que serán de vital importancia manejarlas adecuadamente para mejorar la condición o el estado de salud en que se encuentra el paciente; esto genera un estrés que al ser controlado de manera asertiva permitirá llegar a la "expertise" de la que habla Benner en cuanto a la práctica en enfermería ${ }^{34,35}$.

Generalmente los recién graduados, aún teniendo las bases teóricas, no tienen las capacidades suficientes para desafiar una situación que amerite el actuar rápido y responsable, cuando se ven enfrentados al ámbito profesional, ya que no depende de su tutor en ese momento y tiene que ser autónomo en la toma de decisiones. En algunas ocasiones, la experticia que adquiere la enfermera no solo depende de ella y de la integración de sus capacidades sino del tipo de pacientes que maneje y de su especialidad; puesto que cuanto mayor tiempo esté en contacto con algún tipo de paciente, mayor es la capacidad de predecir y de actuar basado en la experiencia ${ }^{36}$. 
La enfermera(o), más que el afán de adquirir experiencia en un área específica, tiene como motivación el reconocimiento empresarial que genera en algún momento determinado, ascensos e incentivos salariales ${ }^{37}$. Sin embargo debe desarrollar competencias especiales de acuerdo con el área especifica de desempeño, según la clasificación dada por Benner ${ }^{25}$ la etapa más alta que puede adquirir es el de "experta(o)". En el campo asistencial se requiere de este profesional para que se desempeñe en áreas específicas, puesto que reúne los conocimientos, habilidades y actitudes determinadas para ese rol. De acuerdo a la revisión realizada, se proponen como ejemplo tres perfiles en los cuales se señalan las diversas características que debe tener la enfermera experta, se describen los atributos que debe tener una enfermera(o) en el cuidado de los pacientes que se encuentran en urgencias, cuidado crítico y pediatría teniendo en cuenta la concepción que tiene Benner de los principales supuestos de enfermería: persona, ambiente, salud y enfermería, que se consignan en la tabla No. $2^{11}$.

Tabla No. 2. Principales supuestos de Enfermería según la Filosofía de Patricia Benner.

\begin{tabular}{|c|c|c|c|}
\hline Enfermería & Persona & Salud & Situación \\
\hline $\begin{array}{l}\text { Es "una relación de } \\
\text { cuidado. Durante el } \\
\text { proceso } \\
\text { interacción se da un } \\
\text { ambiente reciproco } \\
\text { de ayudar y ser } \\
\text { ayudado, cuya } \\
\text { ciencia está basada } \\
\text { en la moral y en la } \\
\text { ética. La enfermería } \\
\text { busca la historia de } \\
\text { los pacientes y de } \\
\text { esa manera indagar } \\
\text { sus antecedentes. }\end{array}$ & $\begin{array}{l}\text { La persona es un } \\
\text { ser que durante su } \\
\text { vida desarrolla } \\
\text { diferentes } \\
\text { características de } \\
\text { personalidad que } \\
\text { se dan por } \\
\text { experiencias } \\
\text { vividas, el cuerpo } \\
\text { y la mente son } \\
\text { independientes, } \\
\text { de tal modo que la } \\
\text { enfermera centra } \\
\text { sus cuidados en el } \\
\text { cuerpo. }\end{array}$ & $\begin{array}{l}\text { Se define como lo } \\
\text { que se puede } \\
\text { percibir, mientras } \\
\text { que estar sano es } \\
\text { la experiencia } \\
\text { humana de la } \\
\text { salud o de la } \\
\text { integridad. }\end{array}$ & $\begin{array}{lr}\text { Hace referencia } \\
\text { al entorno en } \\
\text { donde } \\
\text { encuentra se } \\
\text { cuerpo el } \\
\text { determinado en } \\
\text { momento, a la } \\
\text { interacción que } \\
\text { se da con el } \\
\text { medio y con las } \\
\text { costumbres. }\end{array}$ \\
\hline
\end{tabular}

Fuente: Brykczynski, $2005^{1}$.

\section{La enfermera(o) en la práctica de urgencias}

La enfermera(o) del servicio de urgencias además de tener una formación integral y una serie de conocimientos frente a las situaciones que se presenten debe poseer una serie de habilidades y destrezas que la convierten en un profesional experto generando calidad en la atención y cuidados al paciente. La enfermera de urgencias desempeña un papel importante en este servicio al realizar la valoración inicial logra clasificar, agilizar procesos y determinar la actuación correspondiente que se debe tener frente al paciente ${ }^{38 .}$

La enfermera(o) en el servicio de urgencias debe tener la capacidad de liderar los procesos y brindar soluciones inmediatas, eficaces y asertivas las cuales cumplan con la atención integral al paciente; durante el desarrollo de las actividades de enfermería debe evaluar la pertinencia del servicio prestado o definir correctivos para el alcance del logro, debe asumir responsabilidades asistenciales, y todas las competencias, 
habilidades y actitudes de acuerdo a las necesidades que demandan los pacientes ${ }^{39}$, lo cual se relaciona con Benner ${ }^{25}$ cuando busca que la enfermera tenga capacidades frente a nuevos retos o se destaque en un área determinada, generando el desarrollo de habilidades tanto cognitivas como manuales, lo que hace que se forme como experto; pertenecer al equipo del servicio de enfermería implica responsabilidad, autonomía, rapidez y veracidad ${ }^{25}$.

En la práctica clínica de urgencias la enfermera debe hacer buen uso de su conocimiento, debido a que de su experticia depende la seguridad y en muchas ocasiones la vida del paciente. Una decisión errada puede ser vital tanto para el paciente como para ella. Adicionalmente el desarrollo en esta área amerita de una practica dinámica que involucra el trabajo de todos los demás profesionales, por ello es necesario el desarrollo de otras habilidades como el trabajo en equipo, toma rápida de decisiones acertadas y resolución de conflictos, que dan como resultado una práctica armónica; y lo más importante la calidad de la atención generando satisfacción del paciente. Es de gran importancia reconocer a la enfermera, pues la atención al paciente en esta área produce altos niveles de estrés porque el equipo de trabajo se encuentra bajo la presión de la situación clínica del paciente y la inestabilidad de las condiciones emocionales de la familia. El desarrollo de una práctica tranquila, eficiente, eficaz en un entorno dinámico tendrá un impacto positivo en la calidad de la atención reflejando cambios en la imagen interior y exterior de la institución ${ }^{39}$.

\section{La enfermera en la práctica del cuidado crítico}

En las últimas décadas, el desarrollo tecnológico en salud y el incremento en la expectativa de vida han propiciado el incremento de unidades de cuidado especificas para pacientes en condiciones clínicas críticas, conviertiendolas en un área que demanda enfermeras(o) con un perfil muy especial por el tipo de trabajo que debe desarrollar en el cuidado de los pacientes que se encuentran allí. En el camino que la enfermera recorre para llegar a la "expertise" en un área de cuidados intensivos se deben considerar los criterios por los cuales fue evaluada para ocupar dicho cargo, y como primera medida se debe estudiar el perfil que requiere una enfermera de cuidado critico. Sobre esto, Guevara ${ }^{40}$ señala que "la enfermera que labora en la Unidad de Cuidados Intensivos (UCl) debe poseer un perfil enmarcado en una filosofía integradora que incluya conocimientos afectivos, emocionales, científicos y tecnológicos" ${ }^{40}$. Otro punto crucial es en el momento de la evaluación por habilidades, donde la enfermera demuestra subjetiva y objetivamente que es competente para desarrollar las actividades propuestas en la $\mathrm{UCl}^{41,42}$.

La formación de una enfermera novata en la $\mathrm{UCI}$ requiere de un constante ejercicio entre la teoría y la práctica para que la calidad de la atención no se muestre afectada $^{43}$, y así mismo tendrá que verse involucrado el acompañamiento incondicional por la enfermera experta puesto que viendo las características que requiere la enfermera en la $\mathrm{UCl}$ es en este momento donde estas se aplican. Es por esta razón que la enfermera debe componerse de sabiduría, agilidad e "intuición"; 44 esta última podría considerarse la de mayor importancia, puesto que dará a la enfermera una base sólida de la que podrá apoyarse para intervenir clínica e intensivamente. Gobed y Chassy ${ }^{45}$, plantean que Benner " propone una nueva teoría de la experiencia y la intuición de enfermería, que hace hincapié en la percepción y solución de problemas siendo conscientes de que están íntimamente relacionados" 45 . 
Cada vez que se brinda una atención en salud basada en conocimientos e intervenciones hacia el paciente la enfermera debe reflexionar al respecto ${ }^{46}$ y establecerse así misma metas enfocadas en mejorar dicha atención para llegar a la excelencia en el cuidado crítico. Concluyendo lo anterior, se puede deducir que la enfermera genera diversas actividades en el área intensivista, donde tendrá que hacer notorio su desempeño, el cual se genera cuando la enfermera es hábil y que por su constante entrenamiento y permanencia en el campo es merecedora del distintivo que la identifique como una enfermera intensivista ${ }^{24-47}$, no queda duda que el perfil requerido por la enfermera que cuida pacientes en la unidad de cuidados intensivos debe cumplir con todos los atributos definidos por Benner ${ }^{11}$ para la enfermera experta.

\section{La enfermera en la práctica pediátrica}

La enfermera(o) especializada(o) en el área de pediatría tiene un perfil más complejo comparado con otras areas de desempeño, teniendo en cuenta que su labor va dirigida al binomio niño-familia. El niño percibe la enfermera(o) como una persona amigable, quien debe tener la suficiente experticia para disminuir el efecto negativo que genera la hospitalización y lograr que el hecho de estar enfermo en lo posible no se convierta en una experiencia desagradable para el mismo ${ }^{48}$, permitiendo un proceso de recuperación más rápido ${ }^{49}$. El profesional, adicional a todos los conocimientos y habilidades requeridos para proporcionar cuidado de buena calidad, debe estar calificado para incorporar la lúdica y así generar agilidad en el proceso de recuperación del paciente ${ }^{50}$. Muchas de estas actividades se desarrollan en un orden secuencial: seleccionar el problema, valorar el estado de salud, buscar la causa e identificar las manifestaciones clínicas, proceder a emitir un diagnóstico de enfermería y termina por priorizar y ejecutar los cuidados y las actividades propias ${ }^{51}$. Una de las funciones de la enfermera(o) es ser un líder que mantenga y mejore los canales de comunicación del paciente y familiares con el equipo de salud que genere un ámbito de confianza para el niño enfermo ${ }^{51}$.

En los servicios de pediatría las enfermeras(o) son tradicionalmente evaluadas por los padres y cuidadores, y la práctica está ampliamente utilizada como la base de pruebas de conocimientos de enfermería ${ }^{52}$ El niño debe permanecer preferiblemente acompañado en forma permanente por un cuidador familiar y por ello en ésta área la familia se convierte en un inspector de todas las actividades que ellas realizan; por tanto, el profesional de enfermería no debe separar al individuo de su contexto más inmediato, la familia ${ }^{53}$. Por el contrario, debe estar preparado para enseñarle a ésta última como cuidar correctamente el niño en casa.

Con base en lo anterior y teniendo en cuenta la definición de experta formulada por Benner, en el cuidado asistencial del paciente pediátrico el profesional de enfermería adapta el contexto para un mejor cuidado del niño y mantiene un vínculo directo e inseparable con su familia, generando un desarrollo profesional más extremo ${ }^{54}$ que da como resultado un cuidado humanizado ${ }^{55}$.

\section{RESULTADOS}

La revisión de la literarura mostró cómo los conceptos de la Filosofía de Patricia Benner ${ }^{11}$ son aplicables en el desarrollo profesional de la enfermera(o), a pesar de no encontrarse trabajos que hagan explícita la utilización de la filosofia en las áreas de urgencias, unidad de cuidados intensivos y pediatría, se encuentra que según el perfil 
requerido en estas áreas, en las tres deben tener los atributos que las clasifican como experta(o).

La planeación, ejecución y evaluación del cuidado requiere de competencias del ser, el saber y el saber hacer, son una serie de habilidades que especifican el arte del cuidado enfermero las cuales son propias de cada individuo cuidador. Así, con base en lo expuesto por Benner ${ }^{25}$ se puede denominar la adquisición de habilidades cognitivas, afectivas, comportamentales y conductuales.

El desarrollo de competencias, del ser, el saber y el saber hacer permiten el constante desarrollo y crecimiento no solo profesional sino también personal; Patricia Benner ${ }^{11}$ busca que la enfermera(o) supere una serie de niveles con la finalidad de llegar a la "experticie". Sin embargo, durante este proceso debe enfrentar situaciones complejas, las cuales pretenden que el profesional cree habilidades como la intuición. Asimismo, en los campos clínicos la enfermera(o) debe reunir características propias de un experto, puesto que de acuerdo al área de desempeño, debe tener la capacidad de desarrollar un plan de atención particular donde brinde un cuidado integral al individuo y a su familia; no solo para el período en el cuál se encuentra en el servicio específico sino también para su egreso.

En los últimos cinco años no se encontró, mucha literatura referente a la aplicación de Patricia Benner ${ }^{11}$, en la formación de los profesionales de Enfermeria, lo cual llama la atención, teniendo en cuenta el auge que ha tenido este periodo la formación por competencias.

\section{CONCLUSIONES}

Con base en los principales supuestos de esta enfermera, se concluye que el paciente es siempre inspirador de cuidado, es el actor de mayor importancia en el proceso de enfermería, es a partir de la identificación de sus necesidades que se elabora, aplica y evalúa el cuidado de enfermería.

Benner ${ }^{25}$ concibe la persona como un ser integral teniendo en cuenta características como la construcción del "yo" que se desarrolla con la experiencia interactiva con los demás, es allí donde se observa al individuo como ser biosicosocial el cual está involucrado en el desarrollo de actividades internas y externas las cuales establecen sus necesidades. A partir de esto, el profesional de enfermería debe actuar con cada ser humano de manera diferente, integrando todas sus necesidades y no valorándolo como objeto pasivo de cuidado, sino como un ser de cambios continuos.

La Literatura revisada permite inferir que en el ámbito clínico se ve reflejado la adquisición de habilidades de la enfermera ya que allí debe poner en práctica todas sus competencias para contribuir en el proceso de tratamiento, rehabilitación y recuperación de cada individuo que presenta alteraciones de salud. Por lo anterior, se puede observar al profesional de enfermería en diferentes áreas específicas en las que desarrolla y adquiere conocimientos y destrezas que fortalecen su que hacer profesional.

Por tanto la filosofía de Benner ${ }^{25}$ es aplicable a la práctica clínica en la cual la enfermera (o) inicia en el nivel de principiante y en la medida que se enfrenta a situaciones diversas en las cuales aplica los conocimientos adquiridos o busca nueva información que le permita la resolución asertiva de las diferentes problemáticas, va 
pasando por los niveles de Principiante avanzada, competente, eficiente hasta adquirir el nivel de experta mediante la adquisición de habilidades y destrezas que serán ejecutadas en las diversas circunstancias que requieren de una enfermera(o) experta (0).

\section{REFERENCIAS}

1. Guimarães L, Vianade Oliveira L. Formas de aprender nadimensãoprática da atuação do enfermeiro asistencial. REBEn 2005;58(5):586-91.

2. Balasco $\mathrm{E}$. The role of the chief nursing officer in learding the practice: Lessons from the Benner Tradition. Nurs Admin Q 2008;32(2):87-91.

3. Pereira Á, Rivera S. O Cuidado de si como principio ético do trabalho em enfermagem. Texo\& Contexto Enfermagem 2005;14 (001):111-9.

4. Busquets $M$. La ética del cuidar. $V$ congreso nacional de enfermería en ostomías. Ponencia. Disponible en: www.estomaterapia.es/Congresos/.../congreso2004/.../Ponencias/5 (Consultado Abril 13, 2012).

5. Benner P. The Wisdom of Our Practice. American Journal of Nursing 2000; 100(10):99-105.

6. Silva I, Vieira M, Dias S, Isse S,Radûnz V, Atherino E, de Santana ME. Cuidado, autocuidado e cuidado de si: umacompreensão paradigmática para o cuidado de enfermagem. Rev. esc. enferm. USP 2009;43(3):697-703.

7. Massó E, Fernández J, Macías C, Betancourt Y. Valoración de algunas teorías de enfermería y la vigencia en la práctica cubana. Revista Cubana de Enfermería 2008; 24:(3-4).

8. Harrison L, Ray A, Cianelli R, Rivera MS, Urrutia M. Competencias en investigación para diferentes niveles de formación de enfermeras: una perspectiva latinoamericana. Ciencia y enfermería 2005;21(1):59-71.

9. Fernández JE. El significado de la fórmula "ser del comienzo" en la ciencia de la lógica de Hegel. Tópicos 2007;15:99-111

10. Alonso LM, Escorcia I. El ser humano como una totalidad. Salud Uninorte 2003;17: 3-8.

11. Brykczynski K. Del principiante a experta: excelencia y dominio de la práctica de enfermería clínica. En: Modelos y teorías en enfermería Quinta Edición. España: Edide, S.L. 2005:165-79.

12. León R. Enfermería ciencia y arte del cuidado. Rev Cubana Enfermer [online]. 2006,22(4) citado 2012-04-19]. Disponible en: http://scielo.sld.cu/scielo.php?script=sci arttext\&pid=S086403192006000400007\&lng=es\&nrm=iso .

13. Durán MM. La ciencia, la ética y el arte de enfermería a partir de conocimiento personal. Revista Aquichan2005;5(1):86-95.

14. Persegona K.R, Rocha D.L, Lenardt M.H, Zagonel I.P. Conhecimento político na atuação do enfermeiro. Esc. Anna Nery [serial on the Internet].2009 Sep[cited 2012 Feb 09];13(3):p.645-650 .

15. Nelson S. Embodied Knowing? The constitution of expertise as moral practice in nursing. Texto y contexto Enfermagem 2007;16(1):136-141.

16. Aguirre E. Aproximación pragmática a la teoría del juicio moral desde la crítica de Kohlberg. Revista Latinoamericana de Ciencias Sociales, Niñez y Juventud 2009;7(2):1273-99.

17. Peña A. The Dreyfus model of clinical problem-solving skills acquisition: a critical perspective. Medical Education Online, North America, 15, jun. 2010. 
Available at: http://med-ed-online.net/index.php/meo/article/view/4846. Date accessed: 03 Feb 2012 .

18. McAllister M. Stakeholders' views in relation to curriculum development Approaches for Australian Clinical Educators. Australian Journal of Advanced Nursing.2006; 24(2);16-20.

19. Kirschner $\mathrm{P}$, Van $\mathrm{P}$. The design of a study environment for acquiring academic and professional competence. Academic Search Elite 2006;22(2):126 -151.

20. Dummas L, Villenueve J, Chevrier L. A tool to evolutive how to learn from experience in clinical settings. Journal of Nursing Education 2000;39(6):251-58.

21. Ramsburg L. An Initial Investigation of the Applicability of the Dreyfus Skill Acquisition Model to the Professional Development of Nurse Educators. Virginia, Marshall University, 2010.

22. Paranhos VD, Mendes MM. Currículo por competencias y metodología activa: Percepción de estudiantes de enfermería. Revista Latino-Americana de Enfermagem 2010;18(1):109-115.

23. Ayers L. Nursing Practice as Knowledge Work within a Clinical Microsystem. Nursing Practice as Knowledge Work. Connecticut, Yale University, 2008.

24. Robinson K, Eck C, Keck B, Wells N. The Vanderbilt Professional Nursing Practice Program. JONA 2003; 33(9):441-50.

25. Benner $\mathrm{P}$, Spichiger $\mathrm{E}$, Wallhagen $\mathrm{M}$. Nursing as a coring practice from a phenomenological perspective. Stand J Caring Sci 2005; 19:303-309.

26. Consejo Internacional de Enfermeras. La definición de enfermería. [Documento en internet]. 2010 [citado 2011 Noviembre 23]. Disponible en: http://www.icn.ch/es/about-icn/icn-definition-of-nursing/

27. Coelho S, Cestari M, Buss M, Leopardi M, Marten V, Oliveira I. Inserção do cuidado terapêutico na construção do conhecimento da enfermagem. Enferm. glob. [revista en la Internet]. 2010 Feb [citado 2012 Abr 23]; (18): Disponible en: $\quad$ http://scielo.isciii.es/scielo.php?script=sci arttext\&pid=S169561412010000100017\&lng=es . . $\quad$ http://dx.doi.org/10.4321/S169561412010000100017 .

28. Landero E. Identificación de los patrones de conocimiento en un incidente Crítico. Horizonte de Enfermería 2002;15:1-10

29. Muñoz F, Morales J, Torres L. El cine en la enseñanza de estrategias para el afrontamiento de la enfermedad. Revista Venezolana de Información, Tecnología y Conocimiento 2009; 6(2):27-37.

30. Wainwright $P$. Towards an esthetics of nursing. Journal of Advanced Nursing, 2000; 32(3): 750-756.

31. Páez R. La ética de las virtudes y enfermería. Revista Mexicana de Enfermería Cardiológica 2000;8(1-4):60-68.

32. Dunston R, Lee A, Lee A, Matthews L, Nisbet G, Pockett R, Thistlethwaite J, White J. Interprofessional Health Education in Australia: The Way Forward. Sydney: University of Technology, Sydney and The University of Sydney. [Documento en internet]. 2009 [citado 2012 Enero 16]. Disponible en: http://www.rilc.uts.edu.au/pdfs/wayforward.pdf

33. Molina P, Jara P. El saber practico en enfermería. Revista cubana de enfermería 2010; 26 (2): 37-43.

34. Juvé $M$, Huguet $M$, Monterde $D$, Sanmartín MJ, Martí $N$, Cuevas $B$, de la Fuente C, Alvareze G. Marco teórico y conceptual para la definición y evaluación de competencias del profesional de enfermería en el ámbito hospitalario. Parte I. Nursing 2007;25 (4):56-61.

35. Benner P, Sutphen M, Kahn V, Day L. Formation And Everyday Ethical Comportment. American Association of Critical-Care Nurses 2008;17: 473-476. 
36. Benner P. Using the Dreyfus Model of Skill Acquisition to Describe and Interpret Skill Acquisition and Clinical Judgment in Nursing Practice and Education. Bulletin of Science, Technology \& Society 2004; 24 (3):188-199.

37. Hargreaves L, Halamak L, Nichols A, Shanks S. A Handoff Report Card for General Nursing Orientation. JONA 2010;40(10): 424-431.

38. Fernández E, Montesinos S, de Miguel MJ, Alié M. Papel de enfermería en el triage de urgencias en atención primaria. Aten Primaria 2008; 40 (12): 641-645.

39. Toranzo T, Aramburu F. Los profesionales de urgencias y emergencias: perfil actual y posibilidades de desarrollo. Pamplona, 2010; 33(1): 13-16.

40. Guevara B, Zambrano A, Evies A. Una aproximación al perfil de la enfermera intensivista. Revista electrónica de portales médicos. [Documento en internet]. 2007 [citado 2011 Noviembre 23]. Disponible en: www.portalesmedicos.com

41. Pleban K, Janiszewski H. Preceptors' Perceptions of a New Evaluation Tool Used During Nursing Orientation. Journal for nurse's in staff development 2010;26 (3):116-122.

42. Nelson $\mathrm{S}$. The search for the good in nursing? The burden of ethical expertise. Nursing Philosophy 2004;5: 12-22.

43. Arteche J. La enfermera sustituta y su adaptación. Enfermería Global [periódico en Internet]. 2009 [citado 2012 Abr 13]; (15). Disponible en: http://scielo.isciii.es/scielo.php?script=sci arttext\&pid=S1695-

61412009000100017\&lng=pt.

44. Ang BC. The Quest for Nursing Excellence. SMJ 2002;43(10):493-496.

45. Gobed F, Chassy P. Towards an alternative to Benner's theory of expert intuition in nursing: A discussion paper. International Journal of Nursing Studies 2008:136-137

46. Skar R. Knowledge use in nursing practice: The importance of practical understanding and personal involvement. Nursing Education Today 2010;30:132-6.

47. Eigsti J. Graduate Nurses' Perceptions of a Critical Care Nurse Internship Program. Journal for nurse's instaff development 2009; 25 (4): 191-8.

48. Velázquez R, Flores JM, Picallos MD, Albar MJ. Experiencias de niños hospitalizados en unidades de pediatría del Hospital Virgen Macarena. Index de Enfermería 2009;18 (4):243-245.

49. Bartel H. La práctica del profesional enfermero experto en las unidades de críticos desde el análisis de una entrevista bajo la perspectiva de Benner. Index Enferm 2010; 19(1):51-54.

50. Alonso S, Menéndez R, Alvarez L, Castillo C. Formación de recursos humanos en enfermería. Rev Cubana Enfermer 2000;16(1):46-50.

51. Torres J, Urbina $O$. Perfiles profesionales, funciones y competencias del personal de Enfermería en Cuba. EducMedSup 2006; 20(1):47-52.

52. Nelson S, McGuilion M. Expertise or performance? Questioning the rhetoric of contemporary narrative use in nursing. Journal of Advanced Nursing 2004; 47 (6): 631-8.

53. Pedraza A. Aspectos conjuntos profesionales pediatría/Enfermería. El equipo pediátrico de Atención Primaria: aspectos formativos y asistenciales. Rev Pediatr Aten Primaria Supl 2011;20:71-80.

54. Fennig T, Bender J, Colby H, Roell R. Genesis of a profesional development tool for ambulatory pediatric Nursing Practice. The Health Care Manager 2005; 24 (4)369-73.

55. Nelson S. Embodied knowing? The constitution of expertise as moral practice in nursing. Texto \& Contexto Enfermagem 2007;16(1):136-41. 
ISSN 1695-6141

๑ COPYRIGHT Servicio de Publicaciones - Universidad de Murcia 